\title{
Imaging of Plasmas Using Proton Beams Generated by Ultra-Intense Laser Pulses
}

M. Borghesi, D.H. Campbell, R.J. Clarke, M. Galimberti, L.A. Gizzi, M. Haines, A.J. MacKinnon, A. Schiavi, O. Willi

This article was submitted to $6^{\text {th }}$ International Conference on Advanced Diagonstics for Magnetic and Inertial Fusion, Villa Monastero, Varenna, Italy, Septerber 3-7, 2001

U.S. Department of Energy

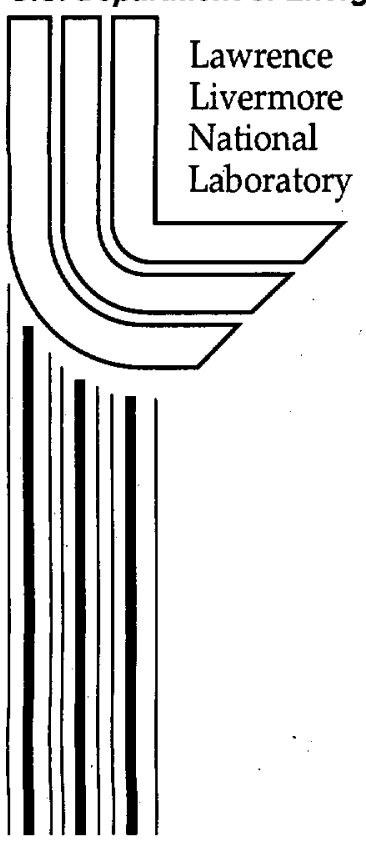

January 15, 2002 


\section{DISCLAIMER}

This document was prepared as an account of work sponsored by an agency of the United States Government. Neither the United States Government nor the University of California nor any of their employees, makes any warranty, express or implied, or assumes any legal liability or responsibility for the accuracy, completeness, or usefulness of any information, apparatus, product, or process disclosed, or represents that its use would not infringe privately owned rights. Reference herein to any specific commercial product, process, or service by trade name, trademark, manufacturer, or otherwise, does not necessarily constitute or imply its endorsement, recommendation, or favoring by the United States Government or the University of California. The views and opinions of authors expressed herein do not necessarily state or reflect those of the United States Government or the University of California, and shall not be used for advertising or product endorsement purposes.

This is a preprint of a paper intended for publication in a journal or proceedings. Since changes may be made before publication, this preprint is made available with the understanding that it will not be cited or reproduced without the permission of the author.

'This report has been reproduced directly from the best available copy.

Available electronically at http://www.doe.gov/bridge

Available for a processing fee to U.S. Department of Energy

and its contractors in paper from

U.S. Department of Energy

Office of Scientific and Technical Information

P.O. Box 62

Oak Ridge, TN 37831-0062

Telephone: (865) 576-8401

Facsimile: (865) 576-5728

E-mail: reports@adonis.osti.gov

Available for the sale to the public from

U.S. Department of Commerce

National Technical Information Service

5285 Port Royal Road

Springfield, VA 22161

Telephone: (800) 553-6847

Facsimile: (703) 605-6900

E-mail: orders@ntis.fedworld.gov

Online ordering: http://www.ntis.gov/ordering.htm

OR

Lawrence Livermore National Laboratory

Technical Information Department's Digital Library

http://www.llnl.gov/tid/Library.html 


\title{
IMAGING OF PLASMAS USING PROTON BEAMS GENERATED BY ULTRA-INTENSE LASER PULSES
}

\author{
M.Borghesi, D.H.Campbell, R.J.Clarke, M.Galimberti, L.A.Gizzi, M.Haines, \\ A.J.MacKinnon, A.Schiavi, O.Willi*
}

\section{INTRODUCTION}

During the interaction of ultraintense laser pulses with plasmas and solid targets, a considerable fraction of the laser energy is deposited into highly energetic charged particles. One of the most exciting results recently obtained in this area of research is the observation of very energetic beams of protons, generated during the interaction of ultraintense short pulses with solid targets. In a number of experiments, performed with different laser systems and in different interaction conditions, protons with energies up to several tens of $\mathrm{MeV}$ have been detected behind thin foils irradiated with high intensity pulses'. In these experiments it was seen that the particle beams are directed along the normal to the back surface of the target, and have a small angular aperture at the highest energies. As proton beams are observed even using target which nominally do not contain hydrogen, protons are thought to originate from hydro-carbon impurities located on the target surfaces ${ }^{2}$ or from bulk contamination of the target. Proposed theoretical models indicate that the protons gain their energy from the enormous electric field ( $\mathrm{MV} /$ micron) set up by laser accelerated fast electrons via space-charge at the back target surface ${ }^{3}$. As protons are easier to accelerate than heavier ions, protons are the dominant component of the ion emission.

The particular properties of the proton beams (small source size, high degree of collimation, short duration, energy dependence on the target characteristics, ease of synchronization with laser pulses) make them very suitable for application as a probe in dense plasmas investigations. Beside the standard radiographic use, i.e. the detection of aerial density variations ${ }^{4}$, proton beams can also be used to detect electric and magnetic fields generated during the interaction of intense laser pulses with plasmas. In this context, proton imaging can be developed as a diagnostic with great potential for Inertial Confinement Fusion (ICF) studies. For example, the possibility of accessing directly electric field distributions in dense plasmas may shed new light on issues as hydrodynamic and electromagnetic instabilities highly detrimental for ICF. The technique has great potential for access to the complex and yet unexplored electric and magnetic field distributions in indirect drive target assemblies. In a Fast Ignitor context, it can contribute to the study of the large transient electric and magnetic fields generated in high-intensity laser-matter interactions. The magnitude and characteristics of these fields are currently the subject of much conjecture in the short pulse, high-intensity plasma physics community.

In this paper the results of a series of experiments ${ }^{5}$ performed at the Rutherford Appleton Laboratory will be presented and discussed, in which the proton source has been applied for the first time as a particle probe in a range of laser-plasma interaction conditions.

\section{EXPERIMENTAL SET-UP AND DIAGNOSTIC ARRANGEMENT}

The VULCAN Nd:glass laser operating in the Chirped Pulse Amplification mode (CPA) was used in the experiment. The main targets (used for the production of the proton beams) were Al foils, $1-2 \mathrm{~mm}$ wide and $3-25 \mu \mathrm{m}$ thick. The $1.054 \mu \mathrm{m}$ CPA interaction pulse, $1 \mathrm{ps}$ in duration, with energy up to $100 \mathrm{~J}$ was focused by an F/3.5 off-axis parabola (OAP) onto the centre of the main target. The incidence was about $15^{\circ}$ off normal. There was the possibility, exploited in the final part of the experimental campaign, of splitting the main CPA pulse in two separate high-intensity pulses, and to focus them employing two separate F/3.5 Off-Axis Parabolas. The focal spot varied between 8 and $10 \mu \mathrm{m}$ in diameter at full width at half maximum (FWHM), containing 30-40\% of the energy, and giving intensities up to $5-7 \times 10^{19} \mathrm{~W} / \mathrm{cm}^{2}$. Heating pulses with duration $0.5-1 \mathrm{~ns}$ were also available, and were used to provide, in some parts of the

\footnotetext{
* M.Borghesi, Department of Pure and Applied Physics, the Queen's University of Belfast, Belfast (UK); D.H.Campbell, A.Schiavi, M.G.Haines, Imperial College of Science, Technology and Medicine, London (UK), O.Willi, Institut für Laser- und Plasmaphysik, Heinrich-Heine-Universität, Düsseldorf (Germany), L.A.Gizzi, M.Galimberti, IFAM-CNR, Pisa (Italy), A.J.MacKinnon, Lawrence Livermore National Laboratory, Livermore (CA, USA)
} 
2

M.BORGHESI ET AL.

experiment, intensities on target in the range $10^{13}-10^{15} \mathrm{~W} / \mathrm{cm}^{2}$. A low-energy fraction of the main CPA pulse was frequency quadrupled and used as a transverse optical probe pulse. A summary description of the beam arrangements used in the various experimental tests performed will be given at the beginning of each experimental section.

The detector employed in the whole campaign consisted of a stack of several layers of radiochromic (RC) film. The film consists of $270 \mu \mathrm{m}$ thick plastic containing a double layer of organic dye, which reacts to ionizing radiation ${ }^{6}$. The equivalent dose of energetic protons stopped in the film can be measured from the changes in optical density undergone by the film, yielding information on the number and energy of the protons. By using them in a stack, each layer of film acts as a filter for the following layers. Since protons deposit energy mainly in the Bragg peak at the end of their range, each RC film layer spectrally selects a narrow range of proton energies. $25-50 \mu \mathrm{m} \mathrm{Al}$ filters were placed in front of the first layer of film giving a minimum detectable proton energy of about $3 \mathrm{MeV}$.

The laser-produced proton beam was characterized in view of its application as a particle beam probe. The protons were produced by focusing the CPA pulse onto the Al foil, and detected by placing the RC film stack at the back of the target, typically at a distance of about $2 \mathrm{~cm}$. It was found that the proton beams have high brightness, typically with $10^{12}$ protons with energy above $3 \mathrm{MeV}$ per shot (at a laser irradiance of the order of $510^{19} \mathrm{Wcm}^{-2}$ ). As observed in previous experiments, the beams were highly directional, propagating along the normal to the back surface of the target with small angular divergence (about $15^{\circ}$ for $10 \mathrm{MeV}$ protons). The response of each RCF layer to high-energy protons has been determined using Monte Carlo modelling of the deposited energy by the particles?. Each film shown responds to ions within an energy band of less than $1 \mathrm{MeV}$. For I $\sim 5-710^{19} \mathrm{~W} / \mathrm{cm}^{2}$ a strong proton signal was observed out to the layer corresponding to proton energy just above $20 \mathrm{MeV}$. The energy deposited within each film layer can be extracted from the absolutely calibrated film. By doing this for each film layer and fitting an exponential energy spectrum, an estimate of the proton energy spectrum and total energy could be extracted from the film data ${ }^{7}$. The mean proton energy of the exponential fit is $3.75 \mathrm{MeV} \pm 0.3 \mathrm{MeV}$ with a total energy of $2.2 \mathrm{~J}$ in an equivalent Maxwellian with a temperature of $2.5 \mathrm{MeV}$. During the experiments the source size of the proton beam was estimated using a penumbral edge method, setting an upper limit of 15-20 $\mu \mathrm{m}$ diameter for the source of $10 \mathrm{MeV}$ protons (there is debate if this is the real or just the apparent source size).

The main reasons making laser-produced proton beams particularly interesting for probing applications are: the small source size, leading to high spatial resolution in imaging applications; the short pulse duration, measured to be a few ps in our conditions; the low divergence and high brightness of the beam; the long stopping range of $10-30 \mathrm{MeV}$ protons, i.e. the possibility of penetrating reasonably thick objects. In addition, high spatial and spectral resolution is guaranteed by an appropriate choice of detector (e.g. RCF stacks), and the proton beam characteristics can be tuned by suitably changing the laser and target parameters. The temporal resolution obtainable with these beams is far beyond the possibilities of beams provided by conventional accelerators. Further, as it will be seen in the following sections, the fact that the beams are not monochromatic is far from being a disadvantage for the proposed applications. Finally their ease of use and synchronization is a clear advantage in laser-plasma and Inertial Confinement-Fusion related experiments. 
IMAGING OF PLASMAS USING PROTON BEAMS GENERATED BY ULTRA-INTENSE LASER PULSES

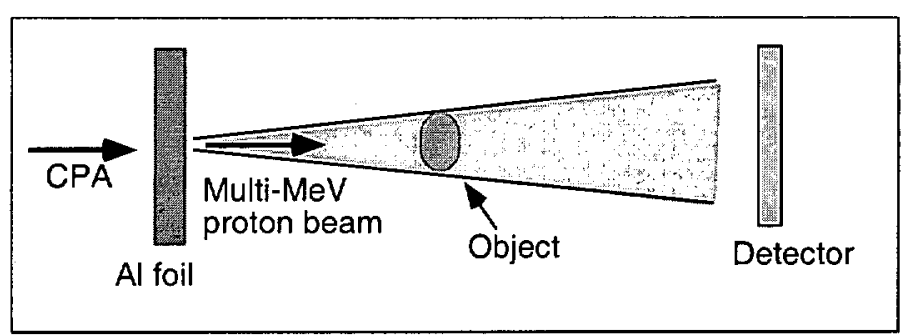




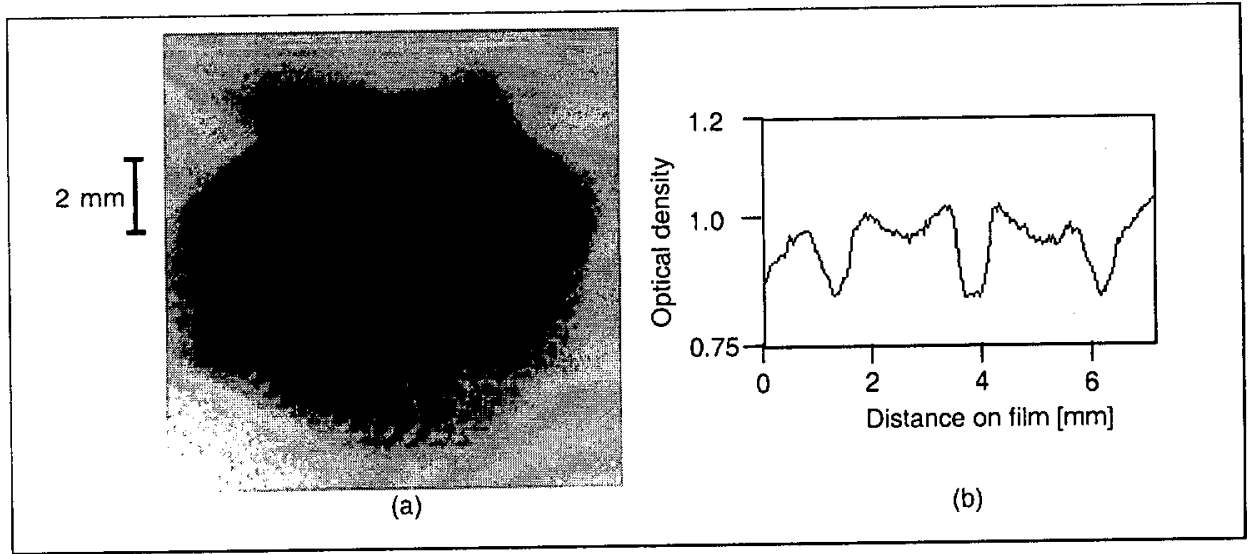




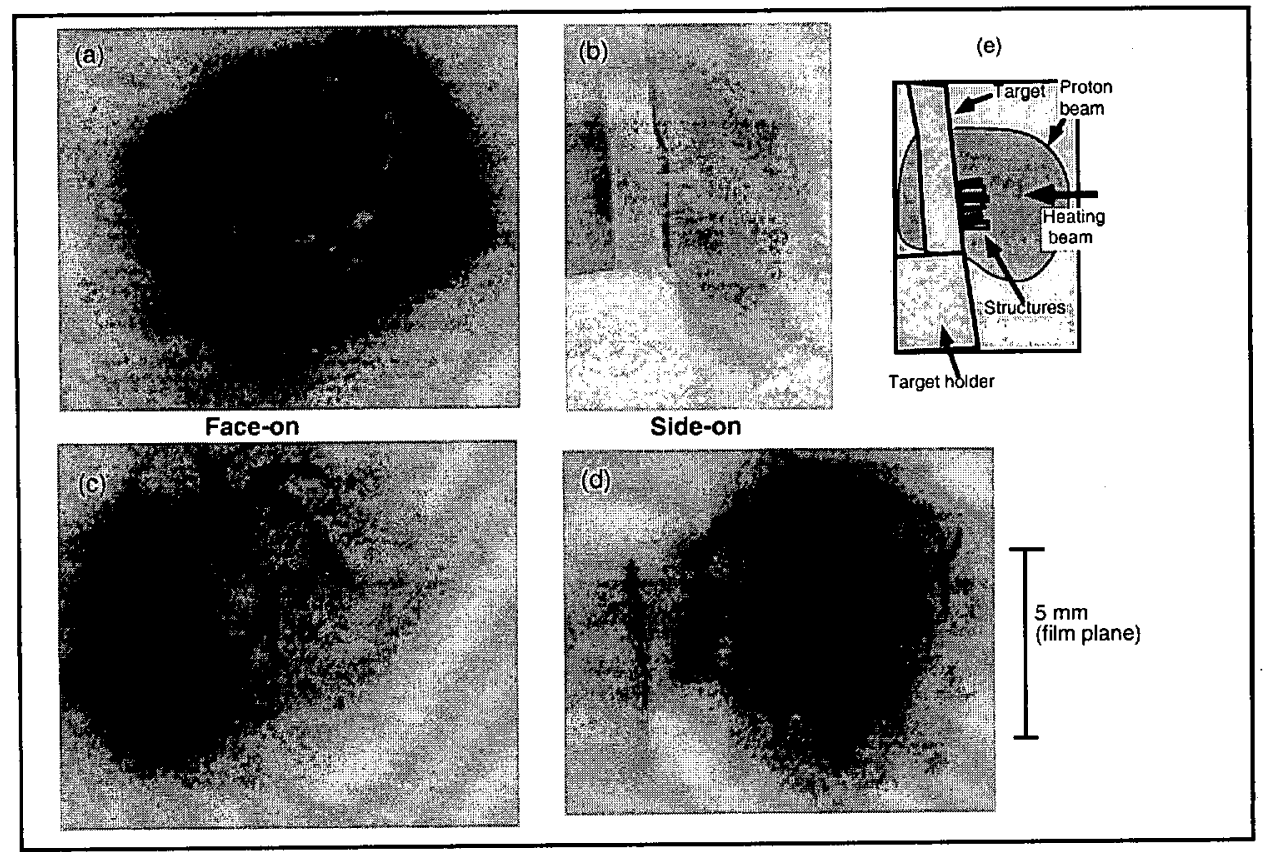

Figure 3 - Face-on and side-on proton images of plasmas produced by laser irradiation of $25 \mu \mathrm{m}$ foils: (a), (b): Heating focal spot : $100 \mu \mathrm{m}$ diameter with no beam smoothing, irradiance: $\mathrm{I} \sim 510^{14} \mathrm{~W} / \mathrm{cm}^{2}$; (c), (d) Heating focal spot: $250 \mu \mathrm{m}$ diameter with beam smoothing (Phase Zone Plates) $\mathrm{I} \sim 10^{14} \mathrm{~W} / \mathrm{cm}^{2}$. (e) Schematic showing the main characteristics of the side-on images.

The proton beam was produced as usual from a $25 \mu \mathrm{m}$ Al foil, and the secondary target also consisted of a $25 \mu \mathrm{m} \mathrm{Al}$ foil. The heating irradiance on target varied between $510^{13}$ and $10^{15} \mathrm{~W} / \mathrm{cm} 2$, with typical delays between the plasma formation and the CPA interaction with the primary target in the range 200-800 ps. When no preformed plasma was produced onto the secondary Al foil, the proton beam was, as expected, unperturbed, except for some charging-up-induced deflection at the edge of the foil. When the secondary foil was laser-heated the situation changed, and, under certain conditions, the presence of the plasma perturbed significantly the intensity distribution of the proton beam. Most importantly the perturbations imposed on the intensity profile of the proton beam appeared to depend strongly on the plasma conditions.

As a matter of fact, changing the irradiation parameters led to striking differences in the modulation pattern superimposed onto the proton beam. An example of this is given in fig. 3, where the RC film images pertaining to different irradiation conditions are shown. The structures observed differ markedly between the case in which no beam smoothing is applied to the beam, and the case in which Phase Zone Plates $(\mathrm{PZP})^{8}$, producing an ordered hot spot structure in the focal spot profile, are employed. In particular side-on images indicate clearly the presence of chaotic structures in the former case, while a regular pattern is observed in the latter. In all cases these structures were only observed within the duration of the heating pulse, and disappeared at later times. We believe that these structures are caused by electric fields present inside the plasma, which are strictly related to the laser intensity distribution. Detailed analysis and modelling is undergoing. 


\section{IMAGING OF ULTRAINTENSE INTERACTIONS}

In part of the experimental campaign, proton imaging was applied to the study of ultraintense laserplasma interactions. These measurements took advantage of the unique possibility of employing two temporally and spatially independent high-power CPA pulses. The second pulse $\left(\mathrm{CPA}_{2}\right)$ was split off the main pulse $\left(\mathrm{CPA}_{1}\right)$ before amplification. Due to set-up constrains, the energy content of each pulse was about $20 \mathrm{~J}$, focusable to intensities in the range $0.5-1.010^{19} \mathrm{~W} / \mathrm{cm}^{2}$. The temporal separation of the two pulses could be varied shot-to-shot. The two beams were focused from different directions with separate $\mathrm{f} / 3.5$ optics. The $\mathrm{CPA}_{2}$ pulse was used to produce a proton beam from an $\mathrm{Al}$ foil, and the proton beam was used to probe, transversely, the interaction of the $\mathrm{CPA}_{1}$ pulse with solid targets.

It should be noted here that, when probing an object undergoing fast changes, the fact that the proton beams are not monochromatic is actually an advantage, as it can be exploited to obtain, in a single shot, information on the temporal history of the object to be probed. In fact, if the object to be probed is situated at a finite distance from the source, protons with different energies will reach the object at different times. By employing a detector that performs spectral selection, the information on the temporal evolution of the target can be retained. In particular, proton imaging was employed to observe the fast evolution of the electric fields following the irradiation of solid targets (wires, foils and micro-balloons) with the CPA, pulse.

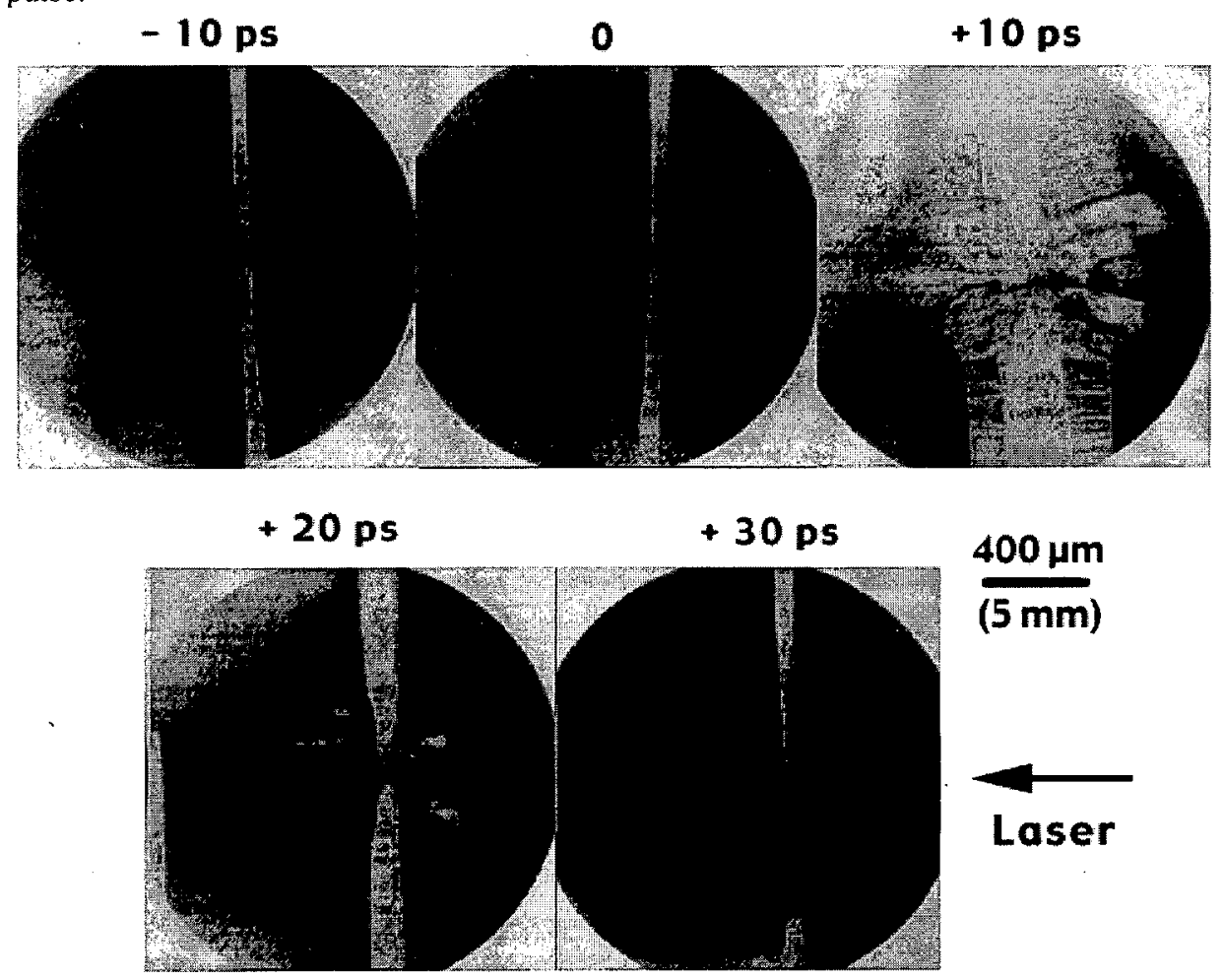

Figure 4 - Temporal evolution of proton images of the interaction zone for different interaction-probing delays. The target was a 50 $\mu \mathrm{m}$ Ta wire and the energy of the protons employed was 6-7 MeV. The delay between $\mathrm{CPA}_{1}$ interaction and probing is indicated above each of the pictures. Spatial scales for both the target and the image plane (between bracket) are indicated. 


\section{IMAGING OF PLASMAS USING PROTON BEAMS GENERATED BY ULTRA-INTENSE LASER PULSES}

Particularly dramatic effects were observed probing the interaction of the $\mathrm{CPA}_{1}$ pulse with small diameter metal wires. Fig. 4 shows proton images of the interaction with a $50 \mu \mathrm{m}$ Ta wire. The RCF layers shown (second layers of the RCF stacks, corresponding to proton energies of 6-7 MeV) have been obtained in separate shots for different $\mathrm{CPA}_{1}-\mathrm{CPA}_{2}$ delays. When the proton probe arrived on target before the $\mathrm{CPA}_{2}$ interaction pulse (e.g., $\mathrm{t}=-10 \mathrm{ps}$, first image in fig.4), only the shadow of the Ta wire, thick enough to slow down the protons is visible, with some small effect visible in the interaction region due to preplasma present ahead of the interaction. However when the probe is coincident with the interaction a dramatic effect is observed, with the protons being deflected away from the wire surface, which charges up due to hot electron expulsion. The charge is seen to decay in a few tens of picoseconds, as filamentary structures (horizontal striations) are seen to appear. The appearance of these structures is consistent with the growth, at the surface of the target, of an electromagnetic heat-flow instability, such as for example the electrothermal instability?.

The instability causes filamentation of the hot current, and as the filaments are magnetized, the effect is imprinted on the proton beam via the radial electric fields present inside the filaments. The onset of pronounced symmetrical horizontal features above and below the interaction axis is also detected. Such structures may be related to the radial expansion of shocks following the interaction, likely presenting nonneutral regions at their leading front.

\section{CONCLUSION}

Proton imaging is a diagnostic with enormous potential for the investigation of fundamental plasma physics problems which were impossible to explore up to now. By using this diagnostic, for the first time the measurement of transient electric fields in dense plasmas has been obtained, determining their evolution on a picosecond scale with micrometric spatial resolution. The data is of great relevance to Inertial Confinement. Fusion both in the conventional and Fast Ignitor approach. Detailed analysis and modelling is presently undergoing.

\section{ACKNOWLEDGEMENT}

The work was supported by an ESPRC grant. We acknowledge the invaluable contribution to the work provided by the Central Laser Facility staff at the Rutherford Appleton Laboratory, and fruitful discussions with Dr. H.Ruhl (General Atomics). 


\section{REFERENCES}

1. E.L Clark et al, Measurements of energetic proton transport through magnetized plasma from intense laser interactions with solids, Phys. Rev. Lett. 84, 670 (2000); R.D.Snavely et al, Intense high-energy proton beams from Petawatt-laser irradiation of solids, Phys. Rev. Lett., 85, 2945 (2000)

2. S.J.Gitomer et $\mathrm{al}$, Fast ions and hot electrons in the laser-plasma interaction, Phys. Fluids 29, 2679 (1984)

3. S. Wilks et al, Energetic proton generation in ultra-intense laser--solid interactions, Phys.Plasmas 8, 542 (2001); A.Pukhov, Threedimensional simulations of ion acceleration from a foil irradiated by a short-pulse laser, Phys. Rev. Lett, 86, 3562 (2001); H.Ruhl et al, Computer simulation of the three-dimensional regime of proton acceleration in the interaction of laser radiation with a thin spherical target, Plasma Phys. Rep. 27, 363 (2001)

4. J.A. Cookson, Radiography with protons, Naturwissenschaften, 61184 (1974)

5. M.Borghesi et al, Proton imaging: a diagnostic for Inertial Confinement Fusion/Fast Ignitor studies, to be published in Plasma Phys. Control. Fusion (2001)

6. W.L.C. McLaughlin et al, Sensitometry of the response of a new radiochromic film dosimeter to gamma radiations and electron beams, Nucl. Instr. Methods Phys. Res. A, 302, 165 (1991)

7. A.J.Mackinnon et al, Effect of plasma scale length on multi-MeV proton production by intense laser pulses, Phys. Rev. Lett, 86, 1769 (2001)

8. T.H.Bett et al, Binary phase zone-plate arrays for laser-beam spatial-intensity distribution conversion, Appl. Opt., 34, 4025 (1995)

9. M.G.Haines, Thermal instability and magnetic field generated by large heat-flow in a plasma, especially under laser-fusion conditions, Phys. Rev. Lett. 47, 918 (1981) 